Supporting Information

for the

Article Entitled

\title{
Multi-state Living Degenerative and Chain Transfer Coordinative Polymerization of $\alpha$-Olefins via Sub-stoichiometric Activation
}

\author{
Authored by \\ Mark A. Wallace and Lawrence R. Sita* \\ Laboratory for Applied Catalyst Science and Technology \\ Department of Chemistry and Biochemistry \\ University of Maryland, College Park, Maryland 20742
}

Details for the polymerization and isolation of atactic poly(1-hexene) (aPH) materials. Supporting data for the characterization of polymeric materials including ${ }^{1} \mathrm{H}$ and ${ }^{13} \mathrm{C}\left\{{ }^{1} \mathrm{H}\right\}$ NMR Spectra and GPC Traces.

\section{Table of Contents}


Table of Contents

Experimental Details

1. General Considerations S3

2. Characterization S3

Polymerization Procedures

Supporting NMR Spectra S5

Supporting GPC Traces S17

References S21

\section{Experimental Details}




\section{General Considerations}

All manipulations with air and moisture sensitive materials were carried out under $\mathrm{N}_{2}$ using standard Schlenk or glovebox techniques. Toluene (Fisher Scientific, 99\%) was dried and deoxygenated by passage over activated alumina and activated copper catalyst (GetterMax 135) and collected under $\mathrm{N}_{2}$ prior to use. Chlorobenzene (Acros Organic, $99 \%$ ) was dried over calcium hydride by refluxing at $130^{\circ} \mathrm{C}$ for three days and distilled under $\mathrm{N}_{2}$ prior to use. Chloroform- $d_{1}$ was purchased from Cambridge Isotopes and used as received. 1-hexene ( $>97 \%$ ) was purchased from TCl Chemicals, dried over $\mathrm{Na} / \mathrm{K}$ alloy and isolated by vacuum-transfer prior to use. Diethylzinc ( $>52 \mathrm{wt} . \% \mathrm{Zn})$ was purchased from Sigma-Aldrich and used as received. $\left[\mathrm{PhNMe}_{2} \mathrm{H}\right]\left[\mathrm{B}\left(\mathrm{C}_{6} \mathrm{~F}_{5}\right)_{4}\right](\mathrm{B} 1)$ was purchased from Boulder Scientific and used as received. Catalyst 1 was synthesized according to published procedures by our group. ${ }^{1}$

\section{Characterization}

Gel Permeation Chromatography (GPC) was used to obtain molecular weight (Mn and $\mathrm{Mw}$ ) and polydispersity index (PDI) of polymers using Viscotek GPCMax equipped with 3 columns (HR 1, HR 3 and HR 4) in a column oven and differential refractometer (Viscotek TDA 302) maintained at $40^{\circ} \mathrm{C}$. Tetrahydrofuran (HPLC Grade) was used as the eluent with a flow rate of $1 \mathrm{~mL} / \mathrm{min}$. Thirteen polystyrene (PS) standards (from Agilent Technologies, $M_{p}=370 \mathrm{Da}-128.7 \mathrm{kDa}$ ) were used for calibration. For GPC sample preparation, $2 \mathrm{mg}$ of dry polymer sample was dissolved in $1 \mathrm{~mL}$ of THF (HPLC Grade). Table $\mathbf{S} 1$ provides the reported and calculated $M_{n}$ and $Đ$ values for the thirteen PS standards.

Nuclear Magnetic Resonance (NMR) Spectroscopy for ${ }^{1} \mathrm{H}$ and ${ }^{13} \mathrm{C}\left\{{ }^{1} \mathrm{H}\right\}$ nuclei was carried out on Bruker AV III HD NanoBay $400 \mathrm{MHz}$ spectrometer equipped with a BBFO probe. Chloroform- $d_{1}$ was used as the solvent for all polymer samples. Spectra were referenced to tetramethylsilane using residual ${ }^{1} \mathrm{H}$ and ${ }^{13} \mathrm{C}\left\{{ }^{1} \mathrm{H}\right\}$ chemical shifts of the deuterated solvents.

\section{Polymerization Procedures}


General Procedure for LCP of 1-hexene. In a $50 \mathrm{~mL}$ round bottom flask, a stirring solution of $0.600 \mathrm{~g}(7.10 \mathrm{mmol})$ of 1 -hexene in $5 \mathrm{~mL}$ chlorobenzene was chilled to $-5^{\circ} \mathrm{C}$. $18.2 \mathrm{mg}(0.04 \mathrm{mmol})$ of 1 was activated with $35.3 \mathrm{mg}(0.044 \mathrm{mmol})$ of B1 in $1 \mathrm{~mL} \mathrm{PhCl} \mathrm{at}$ $-5^{\circ} \mathrm{C}$ and added to the stirring solution. The reaction mixture was stirred for 10 hours at $5{ }^{\circ} \mathrm{C}$ before quenching with $2.0 \mathrm{~mL}$ of acidic methanol $(10 \% \mathrm{v} / \mathrm{v}$ concentrated $\mathrm{HCl})$. Solvent was removed in vacuo and residue was collected in hexanes and passed through a silica plug. Solvent was removed in vacuo and the isolated polymer was dried overnight before GPC and NMR analysis. Details on the amount of the reagents, polymerization time, and polymer characterization are provided in Table 1.

General Procedure for the LCCTP of 1-hexene. In a $50 \mathrm{~mL}$ round bottom flask, a stirring solution of $3.20 \mathrm{~g}(38.0 \mathrm{mmol})$ of 1 -hexene and $49.4 \mathrm{mg}(0.400 \mathrm{mmol})$ of diethylzinc in 5 $\mathrm{mL}$ of toluene was chilled to $-5^{\circ} \mathrm{C} .9 .1 \mathrm{mg}$ of $1(0.02 \mathrm{mmol})$ was activated with $8.0 \mathrm{mg}$ $(0.01 \mathrm{mmol})$ of $\mathbf{B} 1$ in $1 \mathrm{~mL} \mathrm{PhCl}$ at $-5^{\circ} \mathrm{C}$ and added to the stirring solution. The reaction mixture was stirred for 24 hours at $-5^{\circ} \mathrm{C}$ before quenching with $2.0 \mathrm{~mL}$ of acidic methanol $(10 \% \mathrm{v} / \mathrm{v}$ concentrated $\mathrm{HCl})$. Solvent was removed in vacuo and residue was collected in hexanes and passed through a silica plug. Solvent was removed in vacuo and the isolated polymer was dried overnight before GPC and NMR analysis. Details on the amount of the reagents, polymerization time, and polymer characterization are provided in Table 1.

Table S1. Reported and Calculated $M_{n}$ and $\boxplus$ values for PS Standards.

\begin{tabular}{ccccc}
\hline $\begin{array}{c}\boldsymbol{P S} \\
\text { Standard }\end{array}$ & $\begin{array}{c}\boldsymbol{M}_{\boldsymbol{n}}{ }^{\mathbf{a}} \\
(\mathbf{D a})\end{array}$ & $\boldsymbol{\Xi}^{\mathbf{a}}$ & $\begin{array}{c}\boldsymbol{M}_{\boldsymbol{n}}{ }^{\boldsymbol{b}} \\
(\mathbf{D a})\end{array}$ & $\boldsymbol{\Xi}^{\boldsymbol{b}}$ \\
\hline 1 & 387 & 1.15 & 319 & 1.33 \\
2 & 550 & 1.12 & 461 & 1.30 \\
3 & 1,140 & 1.07 & 990 & 1.22 \\
4 & 2,740 & 1.04 & 2,404 & 1.18 \\
5 & 2,910 & 1.04 & 2,581 & 1.17 \\
6 & 4,740 & 1.03 & 4,124 & 1.16 \\
7 & 6410 & 1.03 & 5,374 & 1.14 \\
8 & 10,020 & 1.03 & 8,423 & 1.13 \\
9 & 12,770 & 1.02 & 10,683 & 1.13 \\
10 & 22,230 & 1.02 & 20,080 & 1.11 \\
11 & 28,490 & 1.02 & 25,706 & 1.17 \\
12 & 72,800 & 1.03 & 64,485 & 1.15 \\
13 & 117,600 & 1.06 & 93,277 & 1.22 \\
\hline
\end{tabular}

${ }^{\text {a Reported }} \mathrm{M}_{\mathrm{n}}$ and $\boxminus$ values for the thirteen standards. ${ }^{\mathrm{b}}$ Calculated $\mathrm{M}_{\mathrm{n}}$ and $\boxminus$ values using calibration curve generated from the thirteen standards. 


\section{Supporting NMR Spectra}

A
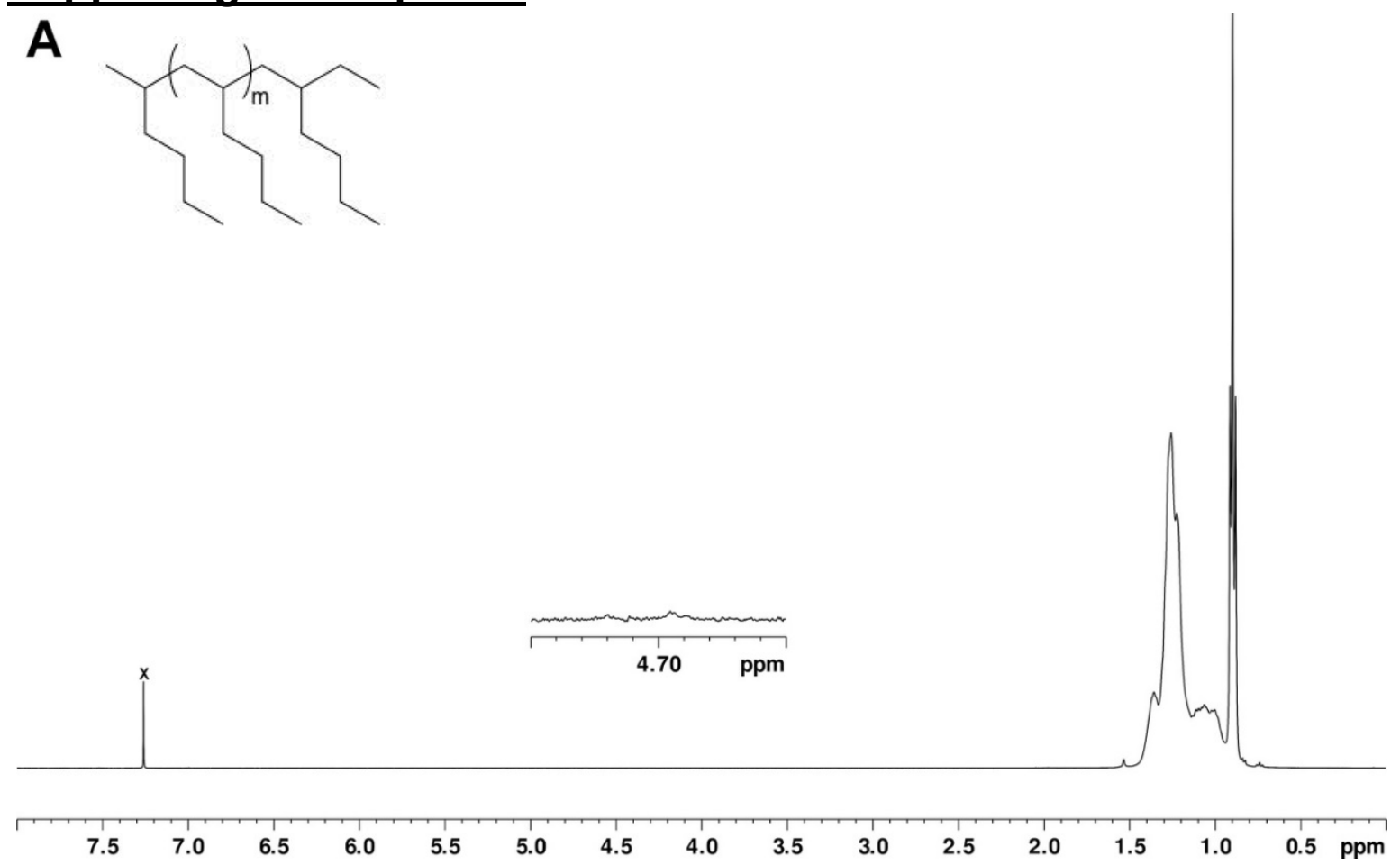

B

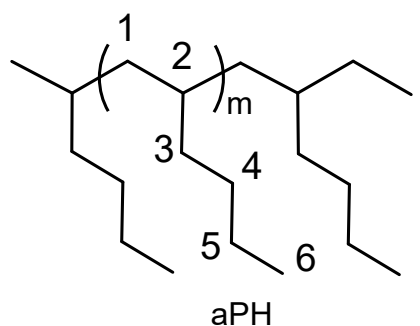

C6

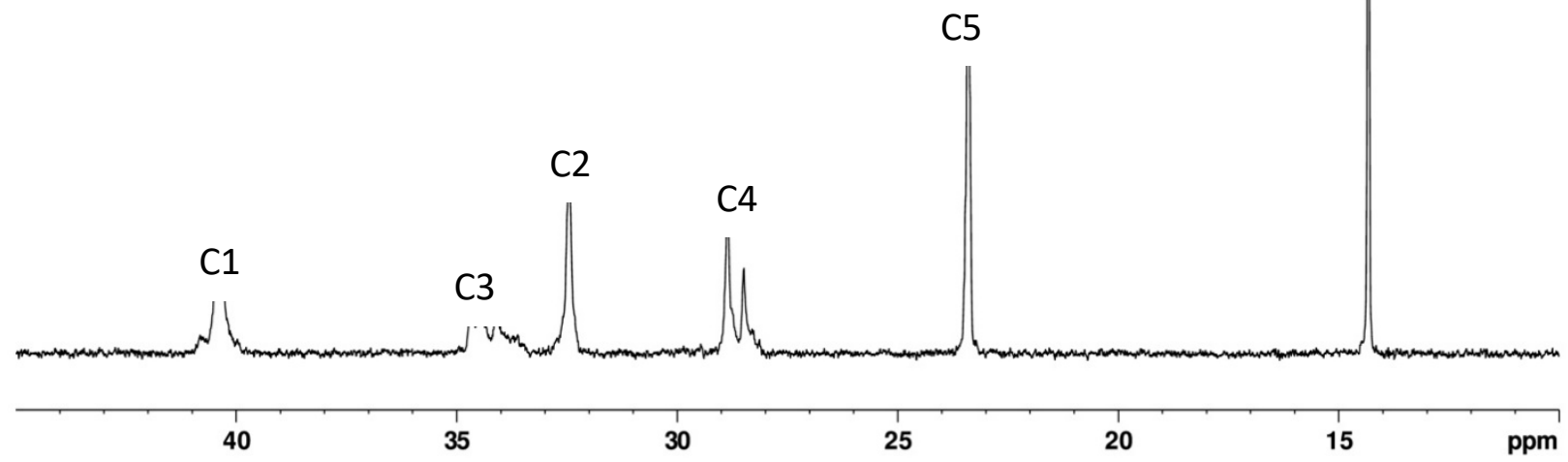

Figure S1. (A) ${ }^{1} \mathrm{H}$ NMR $\left(400 \mathrm{MHz}, \mathrm{CDCl}_{3}, 25^{\circ} \mathrm{C}\right)$ spectrum of aPH from run 1. (B) Partial ${ }^{13} \mathrm{C}$ NMR $(100$ $\mathrm{MHz}, \mathrm{CDCl}_{3}, 25^{\circ} \mathrm{C}$ ) spectrum of aPH from run 1; $\mathbf{X}$ denotes $\mathrm{CDCl}_{3}$ solvent. See ref. 2 for ${ }^{13} \mathrm{C}$ resonance assignments for $\mathrm{aPH}$. 

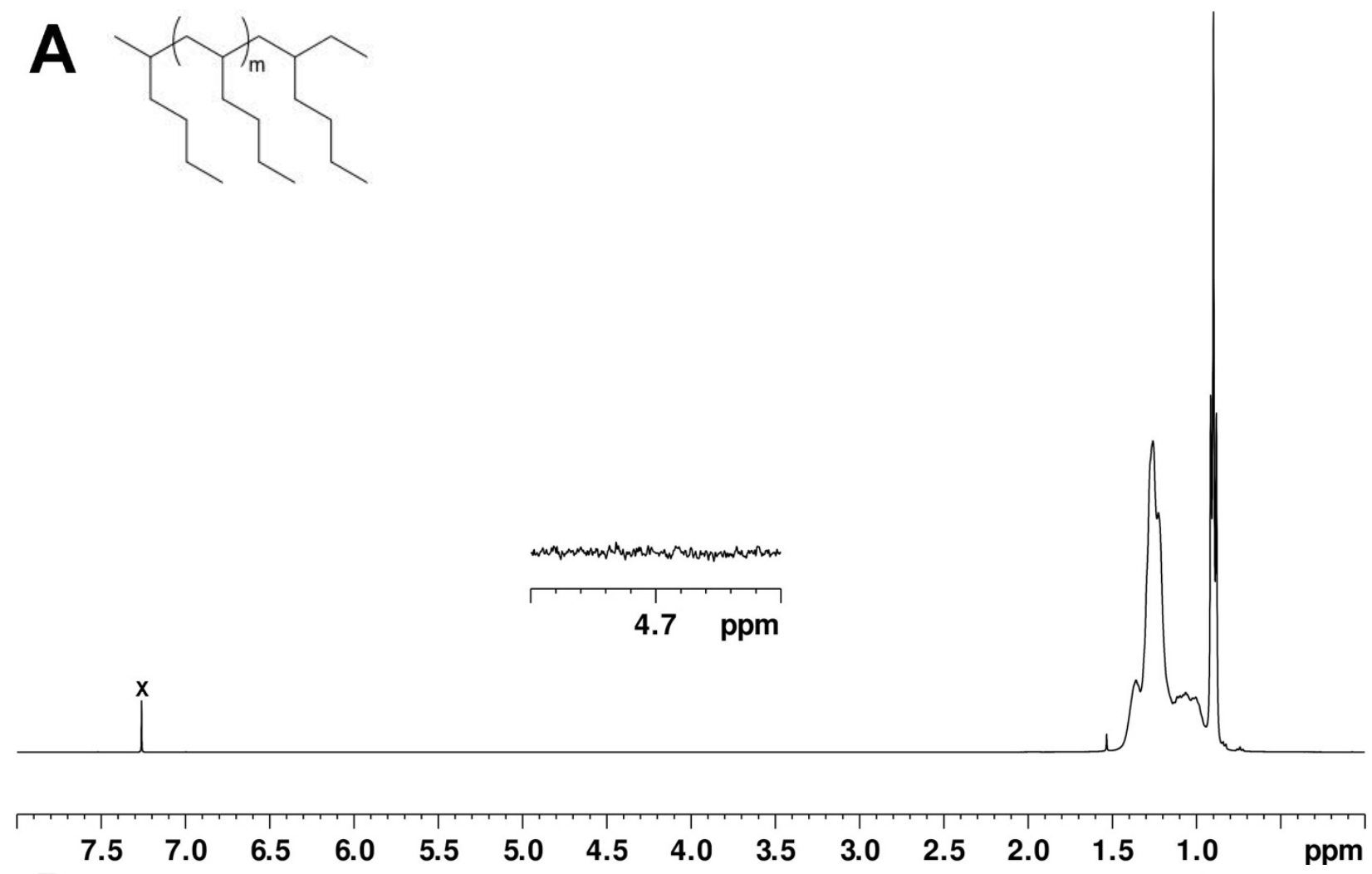

B

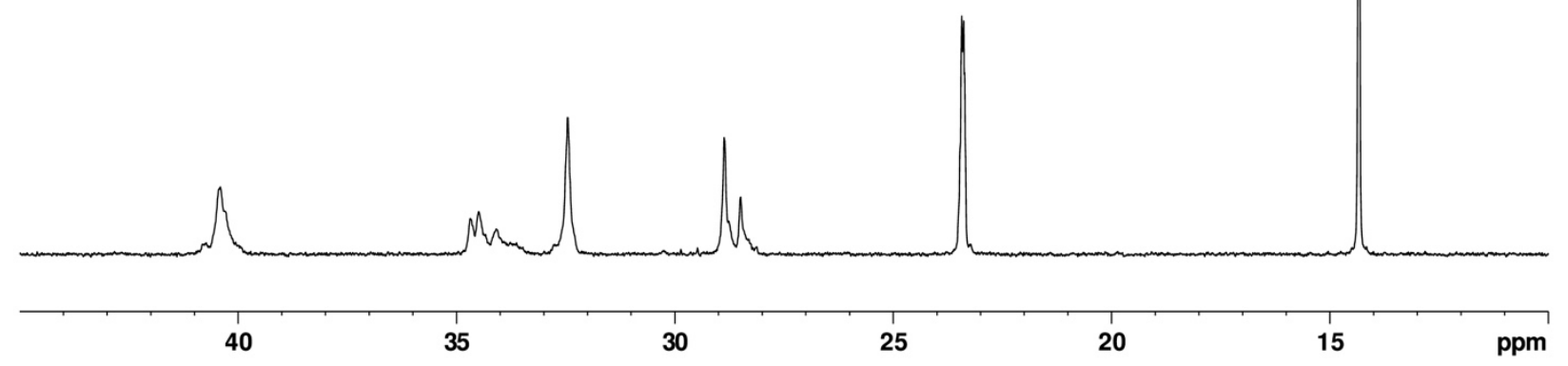

Figure S2. (A) ${ }^{1} \mathrm{H}$ NMR $\left(400 \mathrm{MHz}, \mathrm{CDCl}_{3}, 25^{\circ} \mathrm{C}\right)$ spectrum of aPH from run 2. (B) Partial ${ }^{13} \mathrm{C}$ NMR (100 $\mathrm{MHz}, \mathrm{CDCl}_{3}, 25^{\circ} \mathrm{C}$ ) spectrum of aPH from run 2 ; $\mathbf{X}$ denotes $\mathrm{CDCl}_{3}$ solvent. 

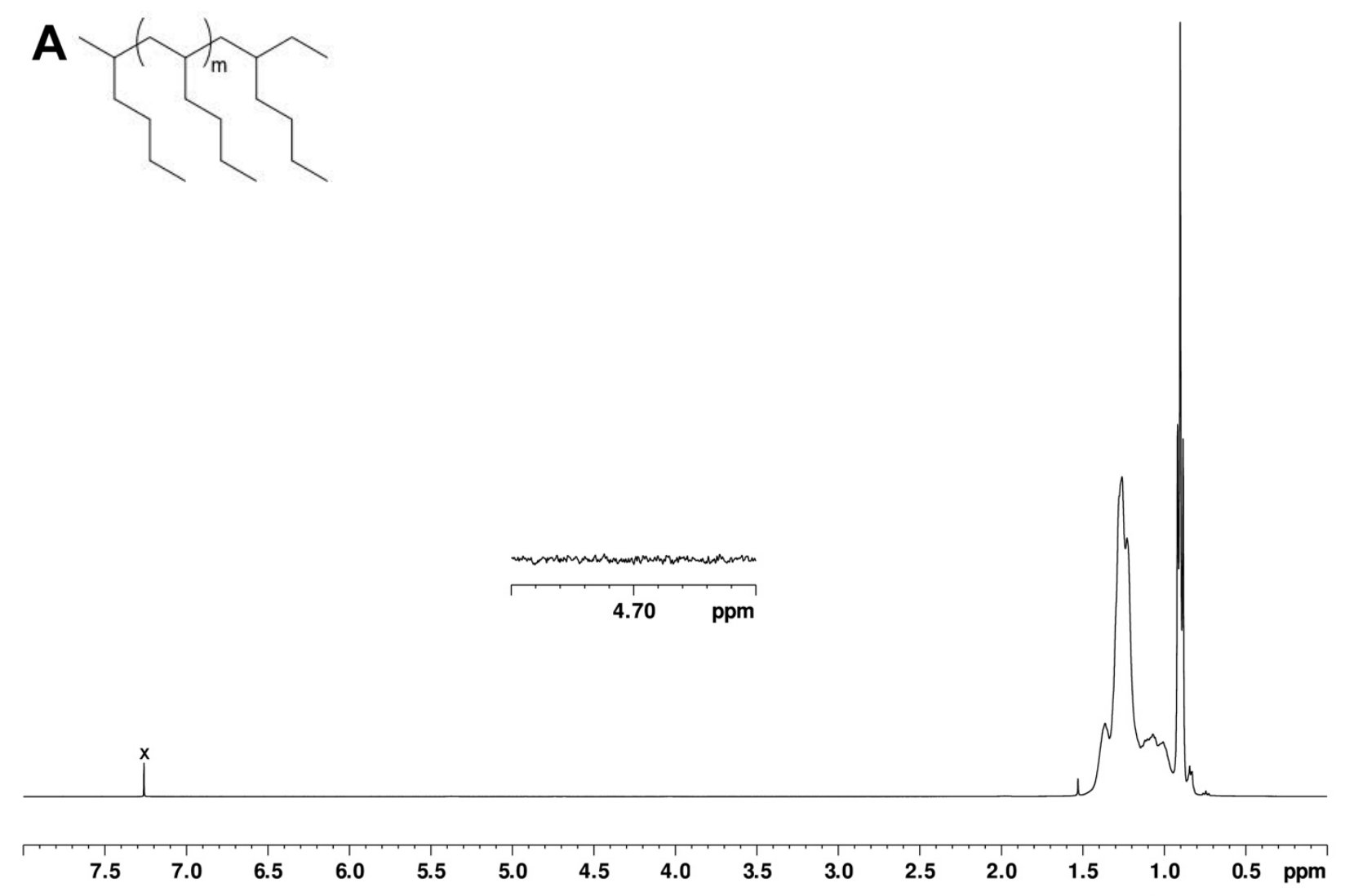

B

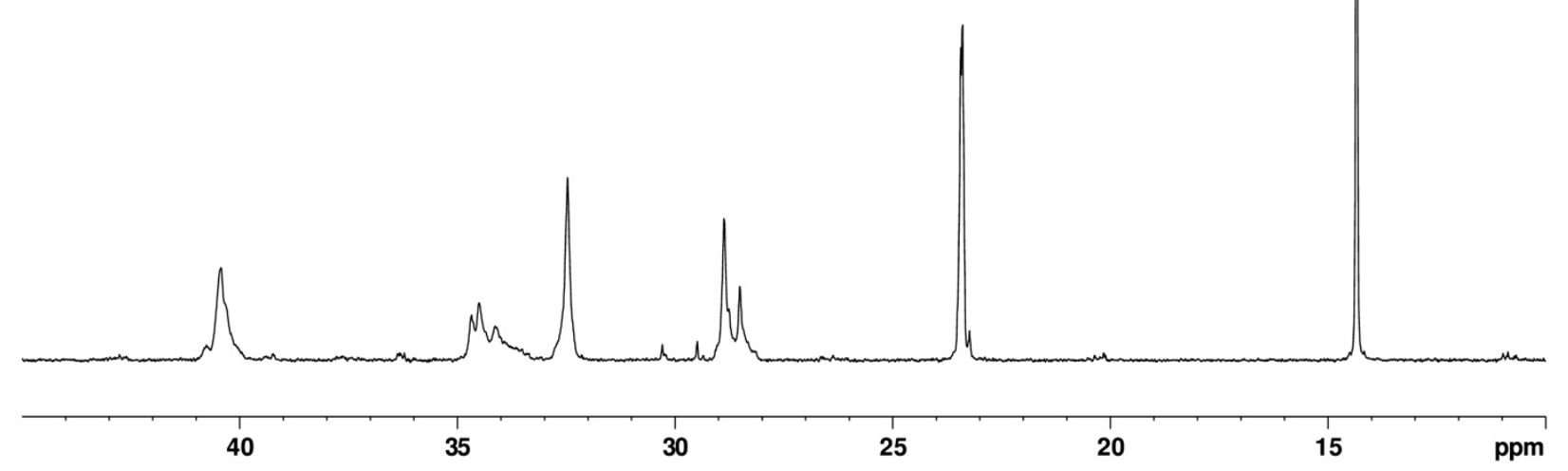

Figure S3. (A) ${ }^{1} \mathrm{H}$ NMR $\left(400 \mathrm{MHz}, \mathrm{CDCl}_{3}, 25^{\circ} \mathrm{C}\right)$ spectrum of aPH from run 3. (B) Partial ${ }^{13} \mathrm{C}$ NMR $(100$ $\mathrm{MHz}, \mathrm{CDCl}_{3}, 25^{\circ} \mathrm{C}$ ) spectrum of aPH from run 3 ; $\mathbf{X}$ denotes $\mathrm{CDCl}_{3}$ solvent. 

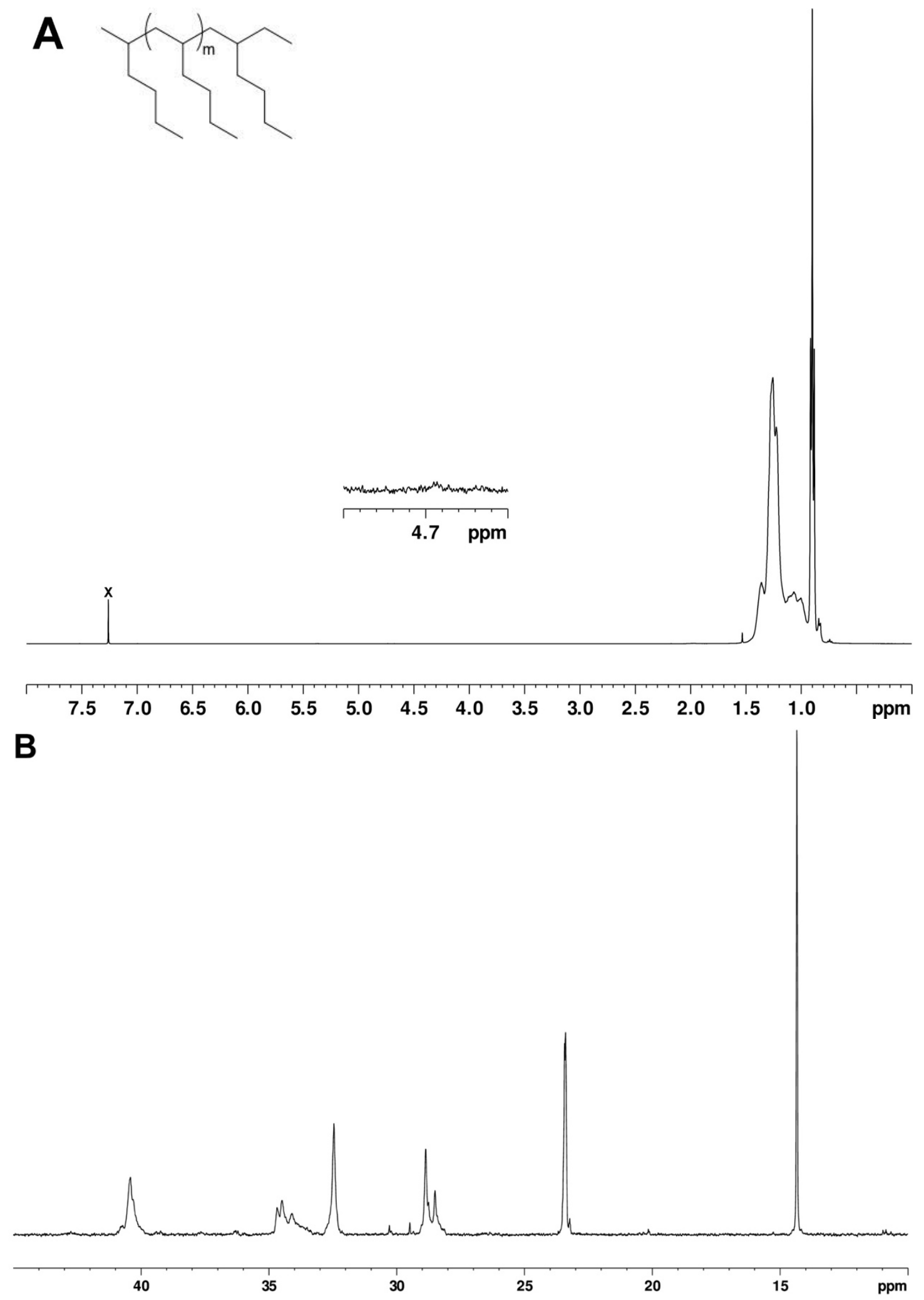

Figure S4. (A) ${ }^{1} \mathrm{H}$ NMR $\left(400 \mathrm{MHz}, \mathrm{CDCl}_{3}, 25{ }^{\circ} \mathrm{C}\right)$ spectrum of aPH from run 4. (B) Partial ${ }^{13} \mathrm{C}$ NMR $(100$ $\mathrm{MHz}, \mathrm{CDCl}_{3}, 25^{\circ} \mathrm{C}$ ) spectrum of $\mathrm{aPH}$ from run $4 ; \mathbf{X}$ denotes $\mathrm{CDCl}_{3}$ solvent. 

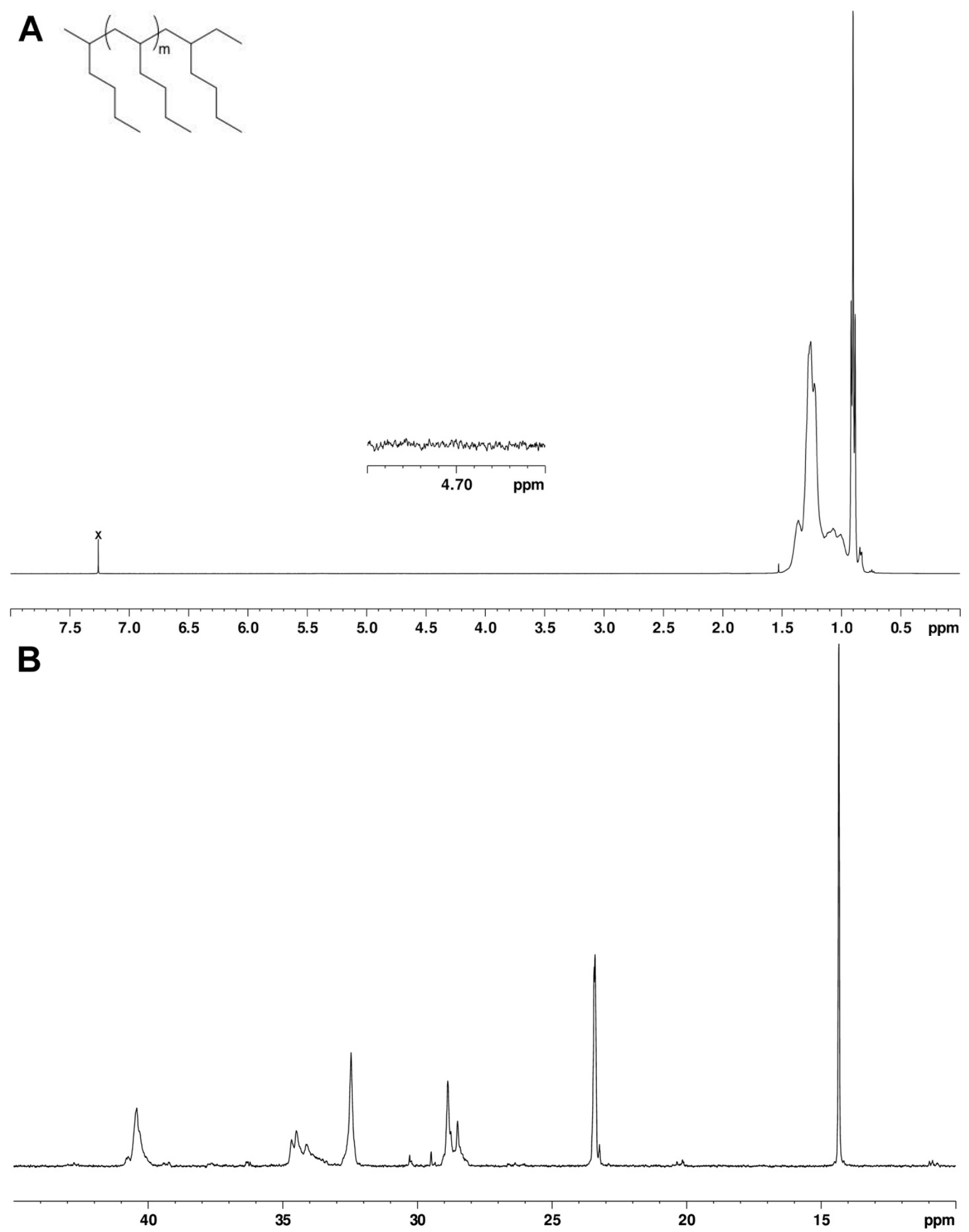

Figure S5. (A) ${ }^{1} \mathrm{H}$ NMR $\left(400 \mathrm{MHz}, \mathrm{CDCl}_{3}, 25^{\circ} \mathrm{C}\right)$ spectrum of aPH from run 5. (B) Partial ${ }^{13} \mathrm{C}$ NMR $(100$ $\mathrm{MHz}, \mathrm{CDCl}_{3}, 25^{\circ} \mathrm{C}$ ) spectrum of aPH from run $\mathbf{5}$; $\mathbf{X}$ denotes $\mathrm{CDCl}_{3}$ solvent 

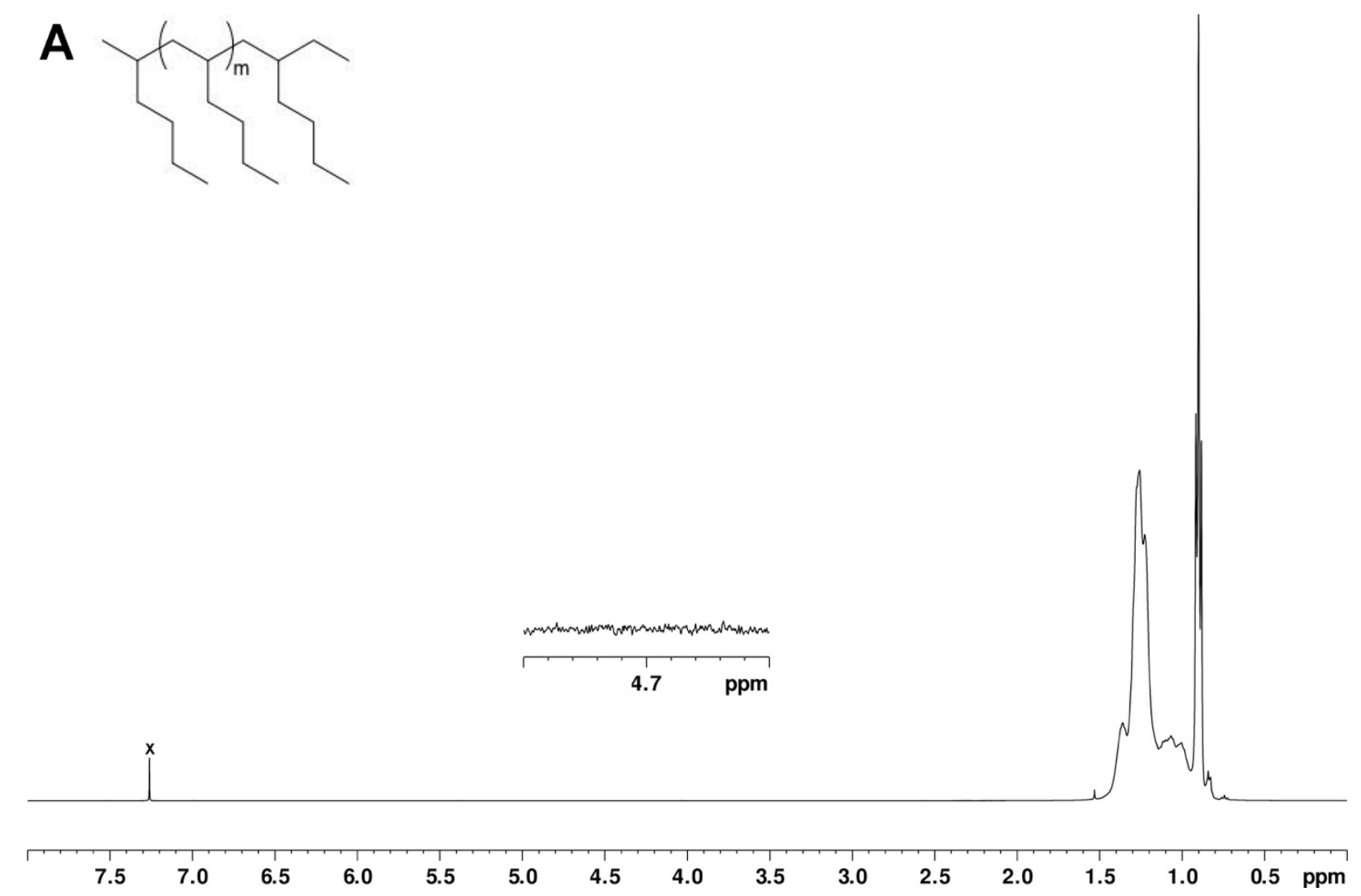

B

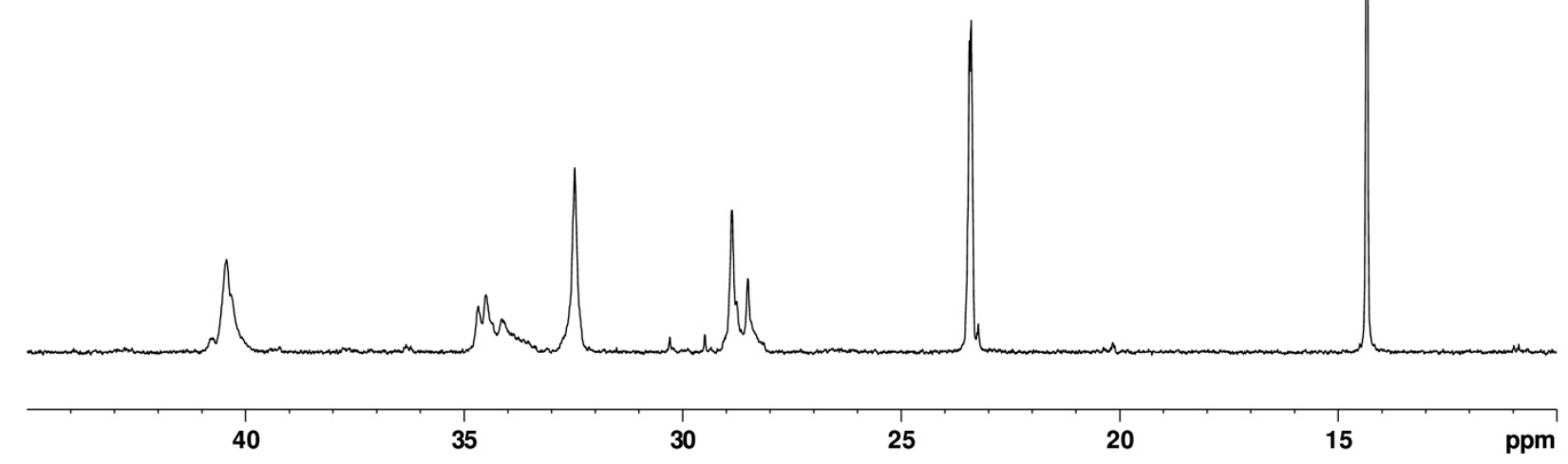

Figure S6. (A) ${ }^{1} \mathrm{H}$ NMR $\left(400 \mathrm{MHz}, \mathrm{CDCl}_{3}, 25^{\circ} \mathrm{C}\right)$ spectrum of aPH from run 6. (B) Partial ${ }^{13} \mathrm{C}$ NMR $(100$ $\mathrm{MHz}, \mathrm{CDCl}_{3}, 25^{\circ} \mathrm{C}$ ) spectrum of aPH from run $\mathbf{6} ; \mathbf{X}$ denotes $\mathrm{CDCl}_{3}$ solvent. 
A
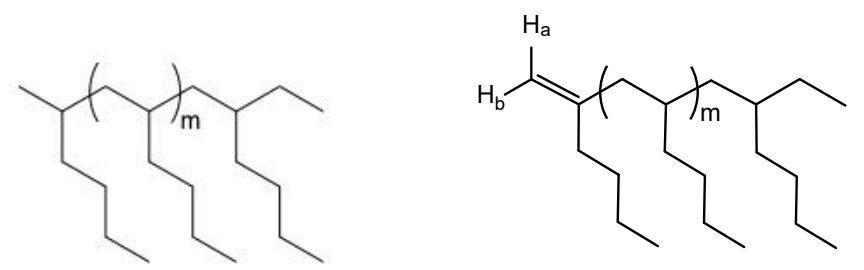

vinylidene from $\beta$-hydrogen transfer

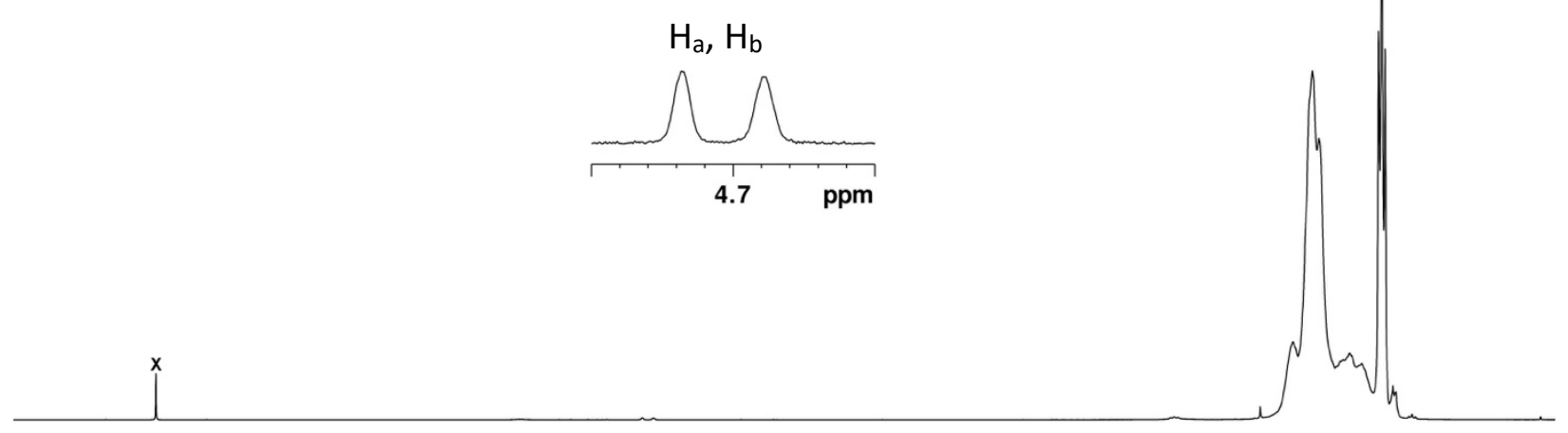

$\begin{array}{llllllllllllllllll}7.5 & 7.0 & 6.5 & 6.0 & 5.5 & 5.0 & 4.5 & 4.0 & 3.5 & 3.0 & 2.5 & 2.0 & 1.5 & 1.0 & 0.5 & \mathrm{ppm}\end{array}$

B

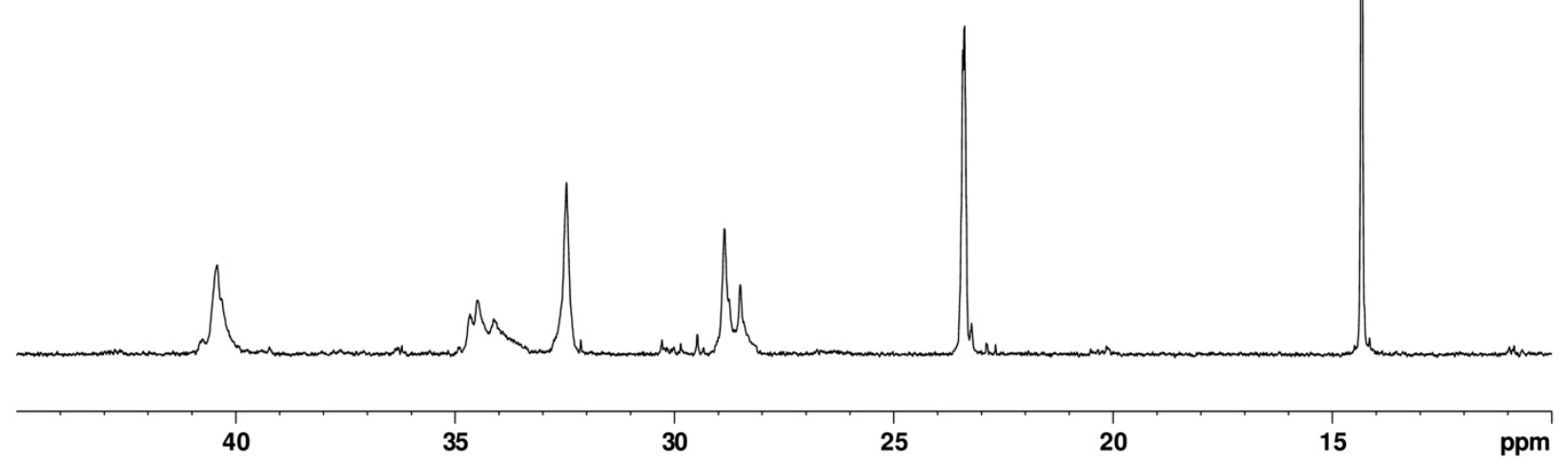

Figure S7. (A) ${ }^{1} \mathrm{H}$ NMR $\left(400 \mathrm{MHz}, \mathrm{CDCl}_{3}, 25^{\circ} \mathrm{C}\right)$ spectrum of aPH from run 7. (B) Partial ${ }^{13} \mathrm{C}$ NMR $(100$ $\mathrm{MHz}, \mathrm{CDCl}_{3}, 25^{\circ} \mathrm{C}$ ) spectrum of aPH from run $7 ; \mathbf{X}$ denotes $\mathrm{CDCl}_{3}$ solvent . 

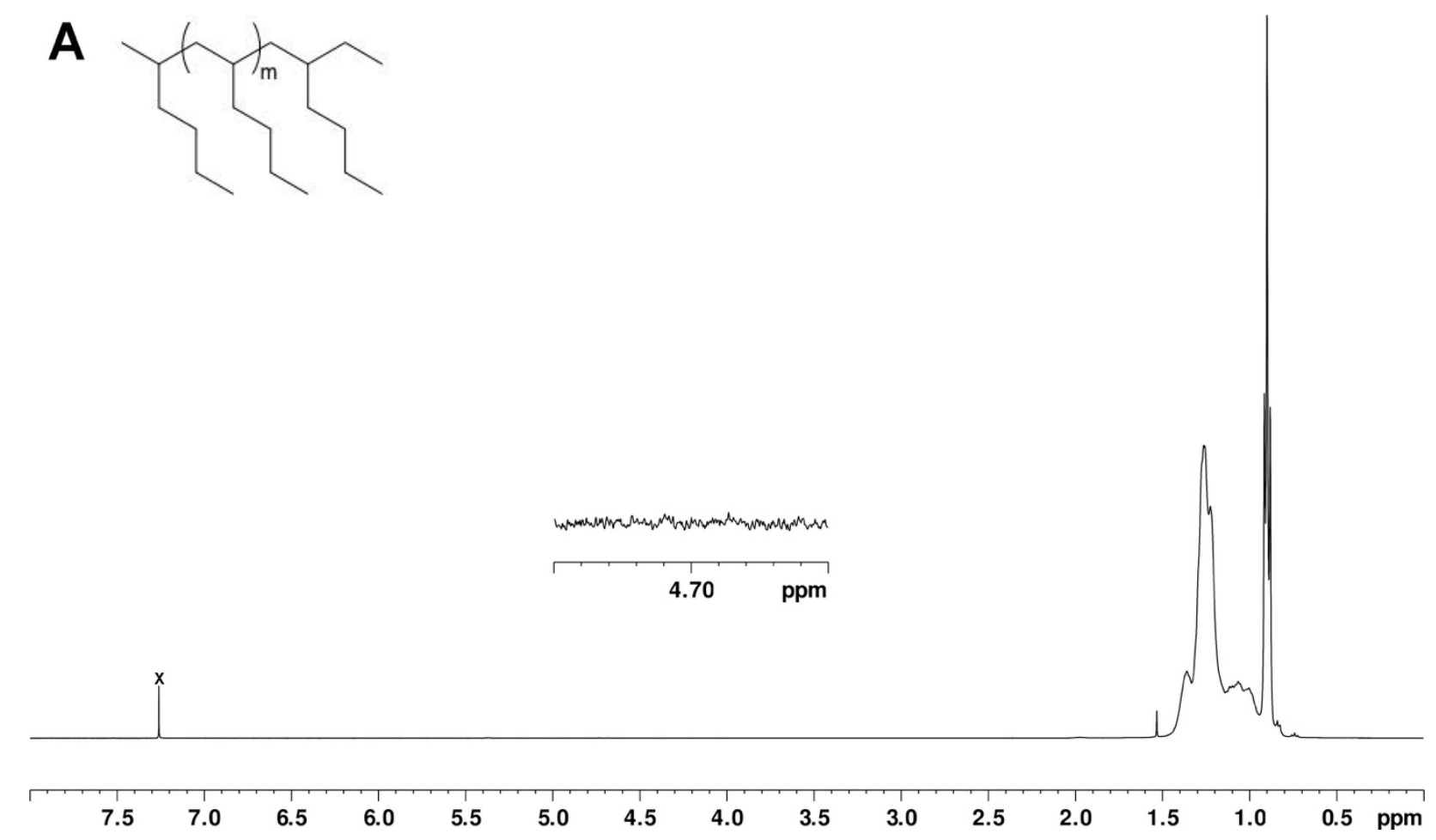

B

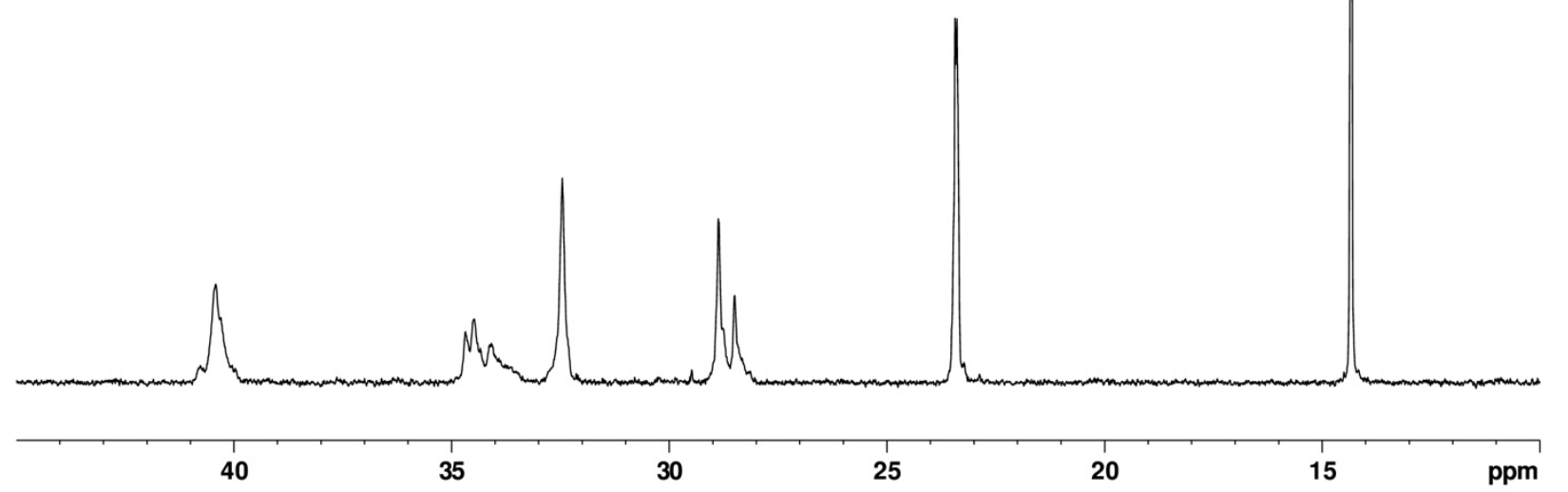

Figure S8. (A) ${ }^{1} \mathrm{H}$ NMR $\left(400 \mathrm{MHz}, \mathrm{CDCl}_{3}, 25^{\circ} \mathrm{C}\right)$ spectrum of aPH from run 8. (B) Partial ${ }^{13} \mathrm{C}$ NMR $(100$ $\mathrm{MHz}, \mathrm{CDCl}_{3}, 25^{\circ} \mathrm{C}$ ) spectrum of aPH from run $\mathbf{8} ; \mathbf{X}$ denotes $\mathrm{CDCl}_{3}$ solvent. 

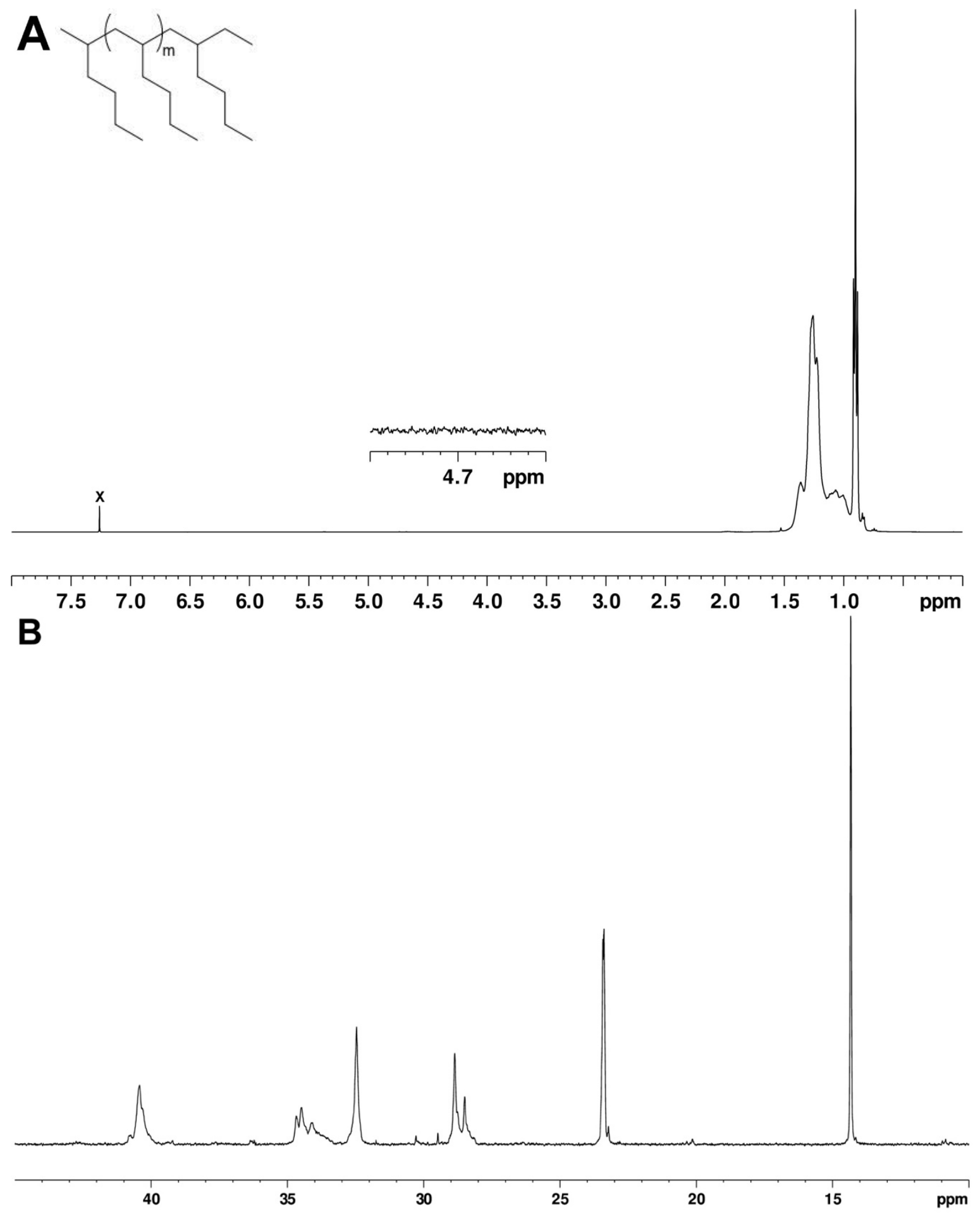

Figure S9. (A) ${ }^{1} \mathrm{H}$ NMR $\left(400 \mathrm{MHz}, \mathrm{CDCl}_{3}, 25^{\circ} \mathrm{C}\right)$ spectrum of aPH from run 9. (B) Partial ${ }^{13} \mathrm{C}$ NMR $(100$ $\mathrm{MHz}, \mathrm{CDCl}_{3}, 25^{\circ} \mathrm{C}$ ) spectrum of aPH from run $\mathbf{9} ; \mathbf{X}$ denotes $\mathrm{CDCl}_{3}$ solvent. 


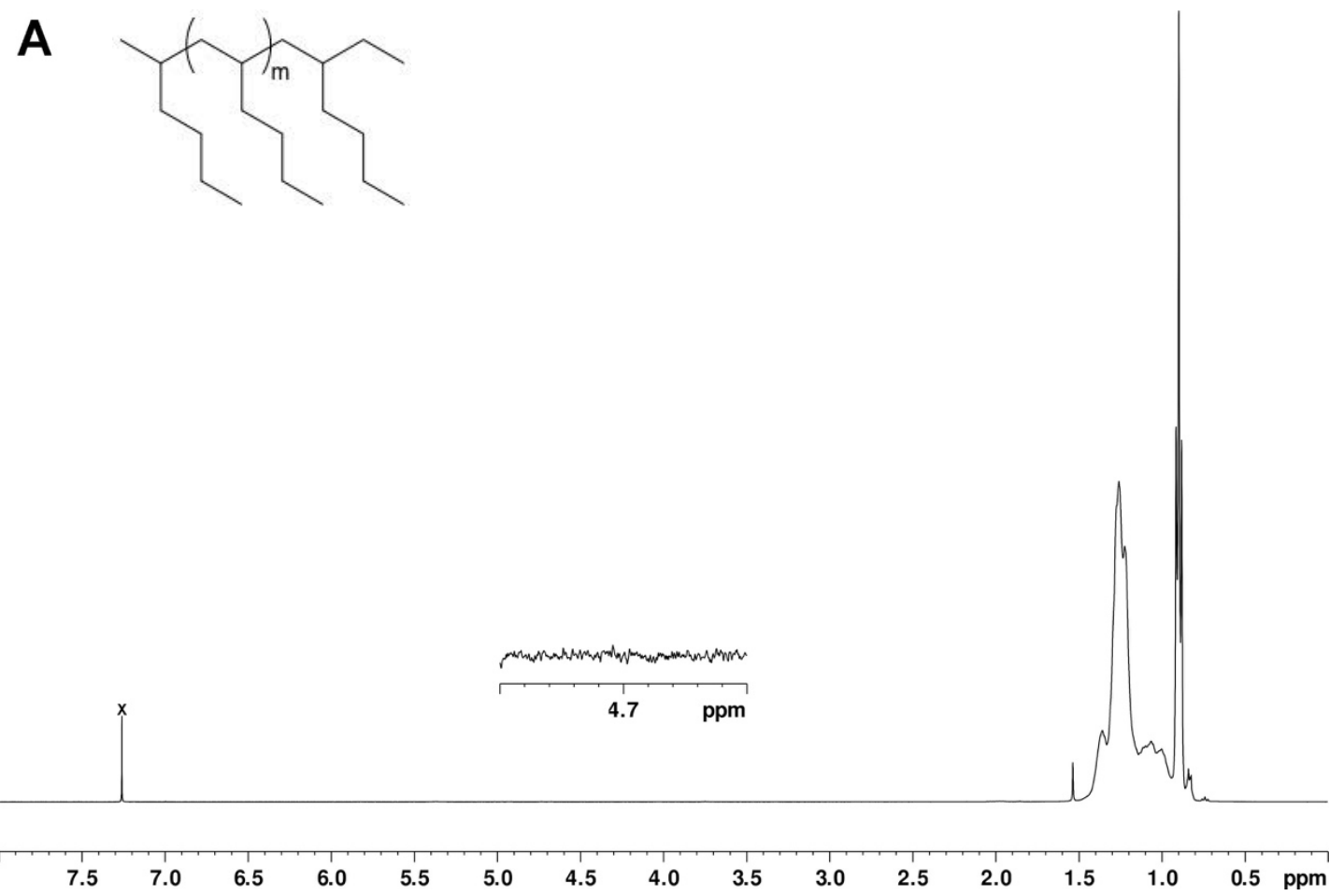

B

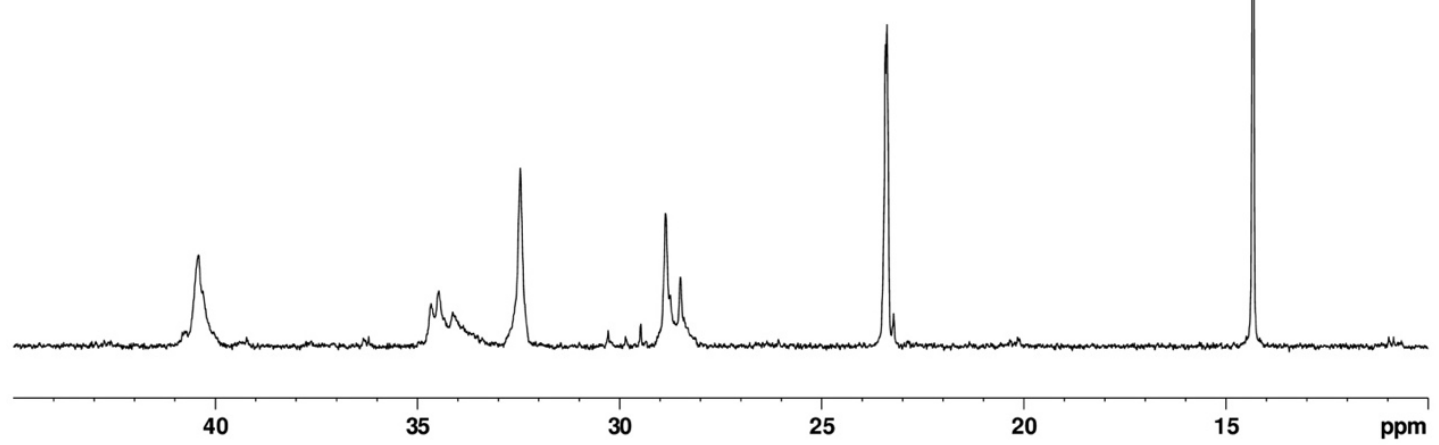

Figure S10. (A) ${ }^{1} \mathrm{H}$ NMR $\left(400 \mathrm{MHz}, \mathrm{CDCl}_{3}, 25{ }^{\circ} \mathrm{C}\right)$ spectrum of aPH from run 10. (B) Partial ${ }^{13} \mathrm{C}$ NMR $\left(100 \mathrm{MHz}, \mathrm{CDCl}_{3}, 25^{\circ} \mathrm{C}\right.$ ) spectrum of aPH from run 10; $\mathbf{X}$ denotes $\mathrm{CDCl}_{3}$ solvent. 

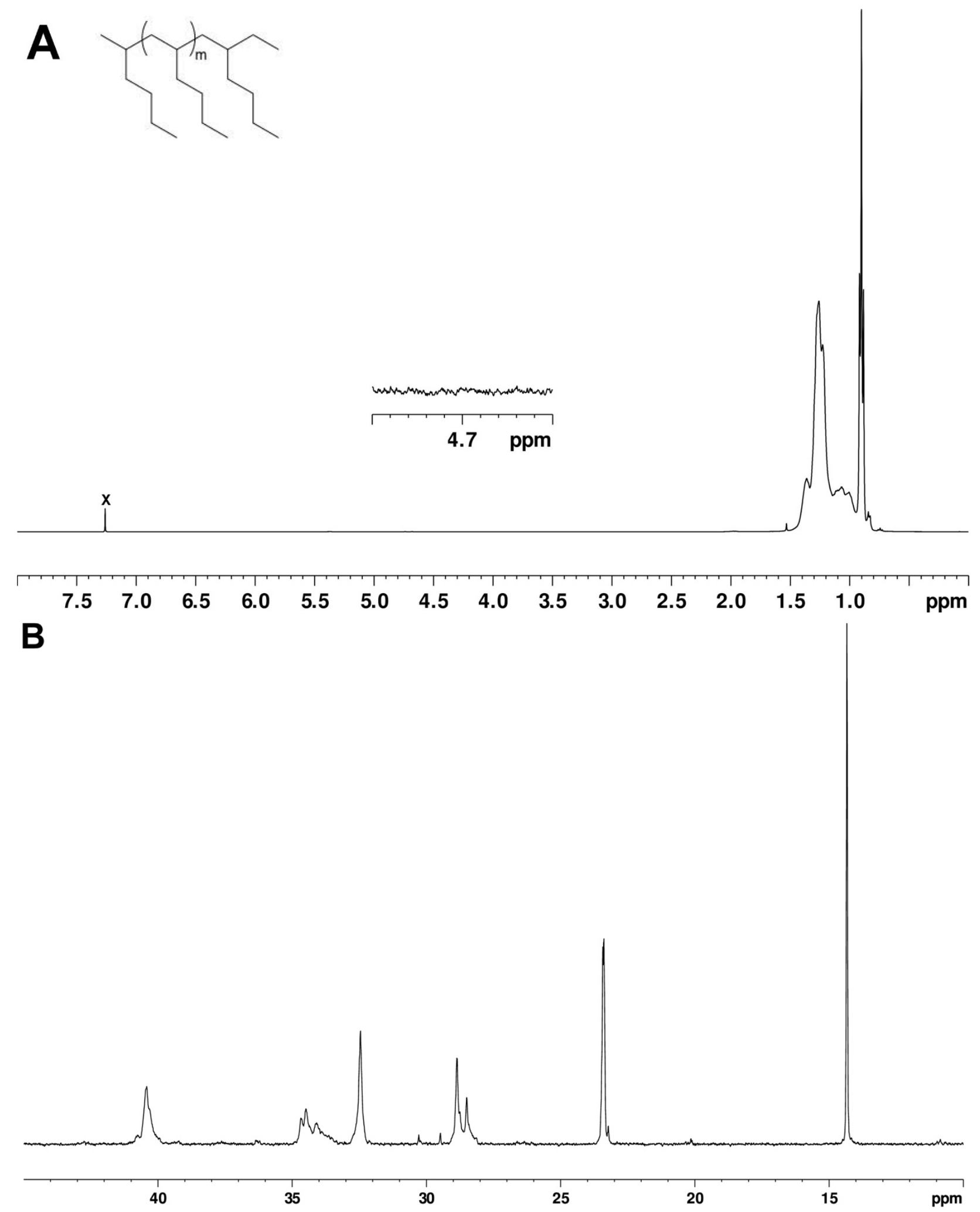

Figure S11. (A) ${ }^{1} \mathrm{H}$ NMR $\left(400 \mathrm{MHz}, \mathrm{CDCl}_{3}, 25^{\circ} \mathrm{C}\right)$ spectrum of aPH from run 11. (B) Partial ${ }^{13} \mathrm{C}$ NMR $\left(100 \mathrm{MHz}, \mathrm{CDCl}_{3}, 25^{\circ} \mathrm{C}\right.$ ) spectrum of aPH from run $11 ; \mathbf{X}$ denotes $\mathrm{CDCl}_{3}$ solvent. 

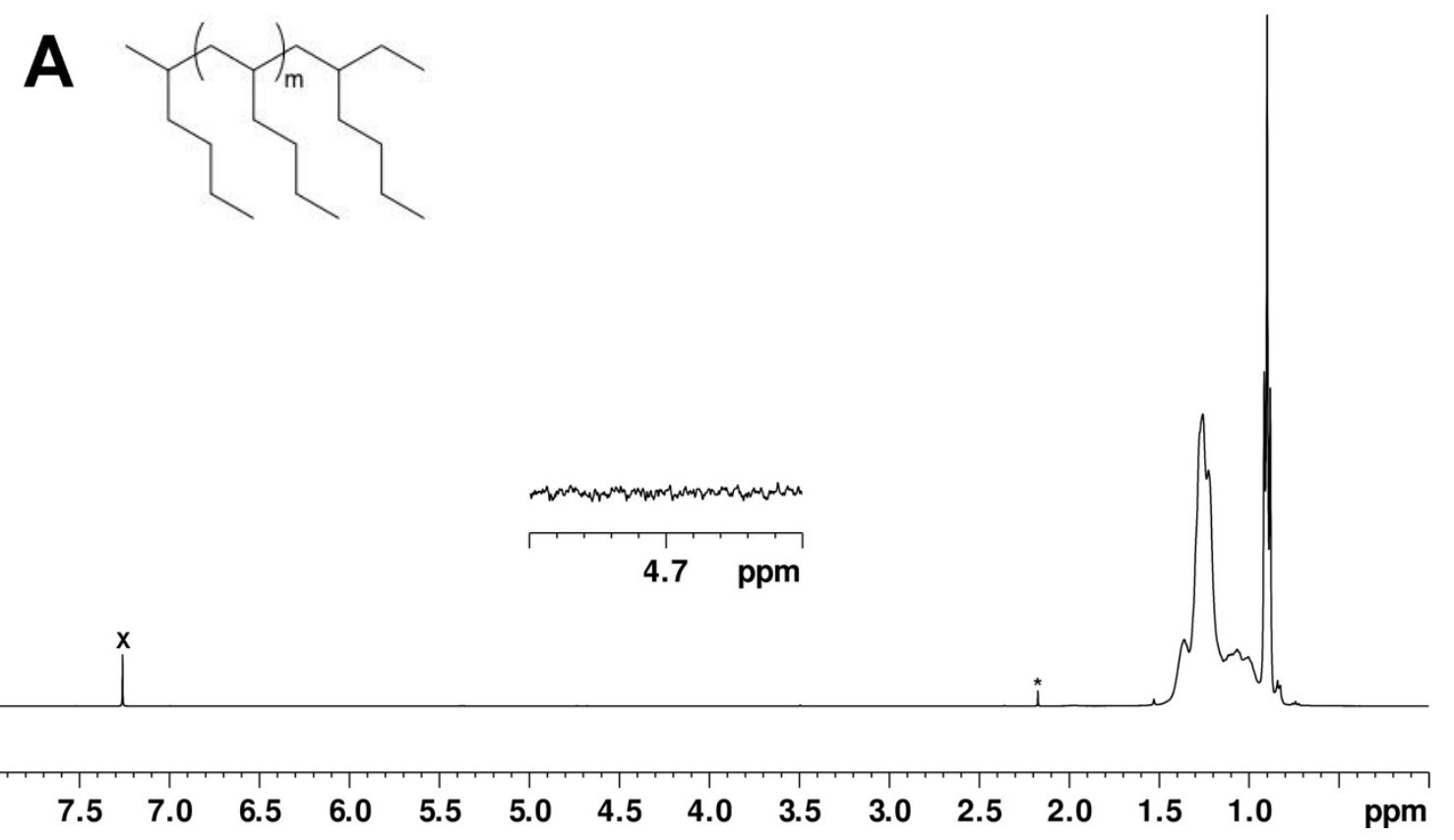

B

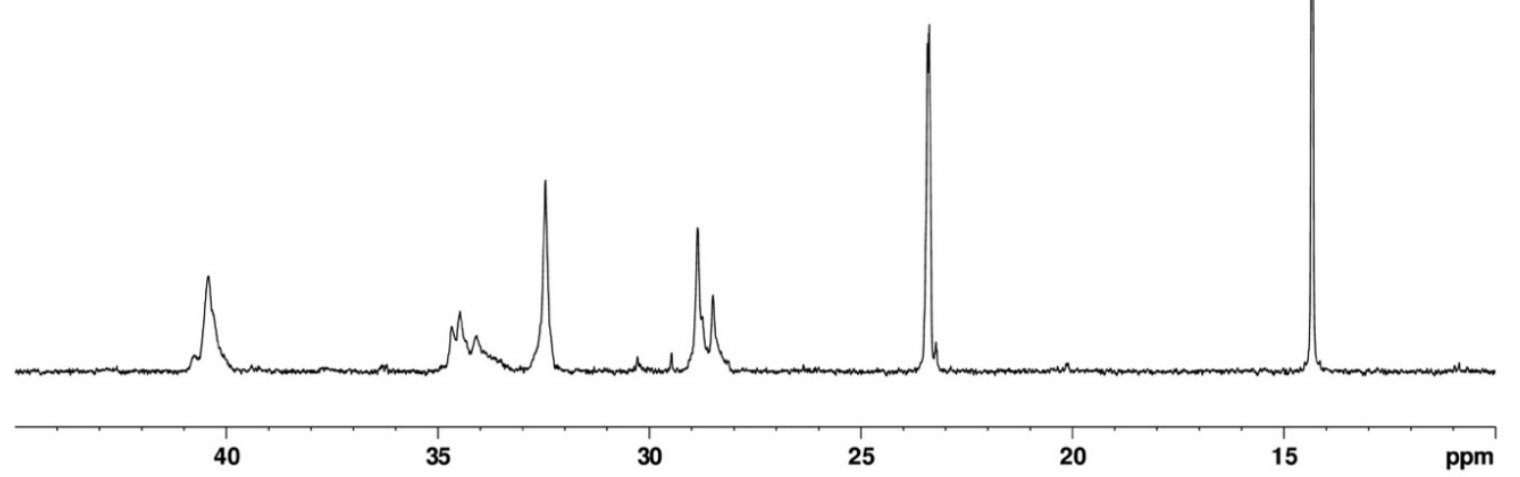

Figure S12. (A) ${ }^{1} \mathrm{H}$ NMR $\left(400 \mathrm{MHz}, \mathrm{CDCl}_{3}, 25^{\circ} \mathrm{C}\right)$ spectrum of aPH from run 12. (B) Partial ${ }^{13} \mathrm{C} \mathrm{NMR}$ $\left(100 \mathrm{MHz}, \mathrm{CDCl}_{3}, 25^{\circ} \mathrm{C}\right.$ ) spectrum of aPH from run $12 ; \mathbf{X}$ denotes $\mathrm{CDCl}_{3}$ solvent / * denotes acetone solvent impurity. 


\section{Supporting GPC Traces}
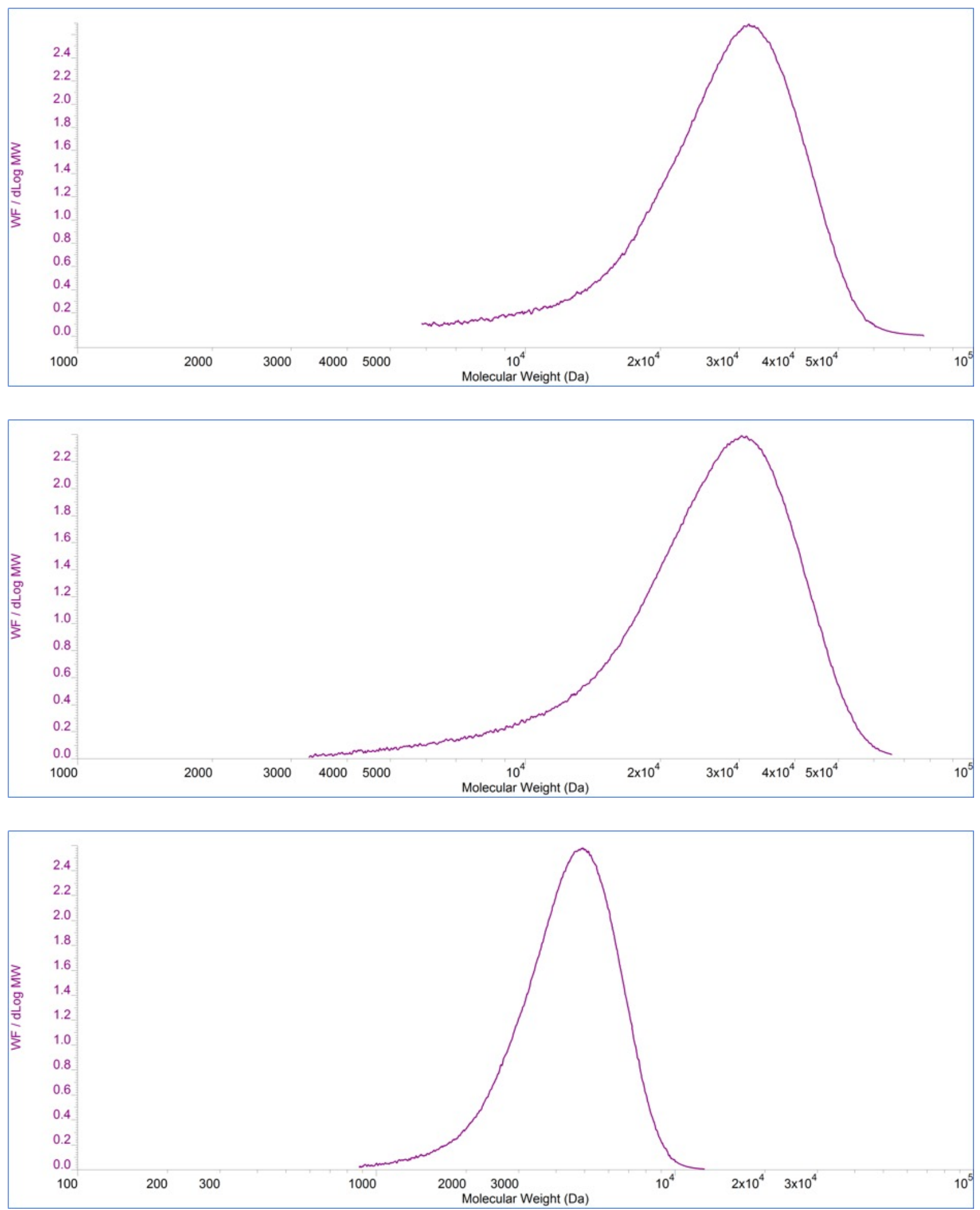

Figure S13. GPC trace of aPH from run 1 (top). GPC trace of aPH from run 2 (middle). GPC trace of aPH from run 3 (bottom). 

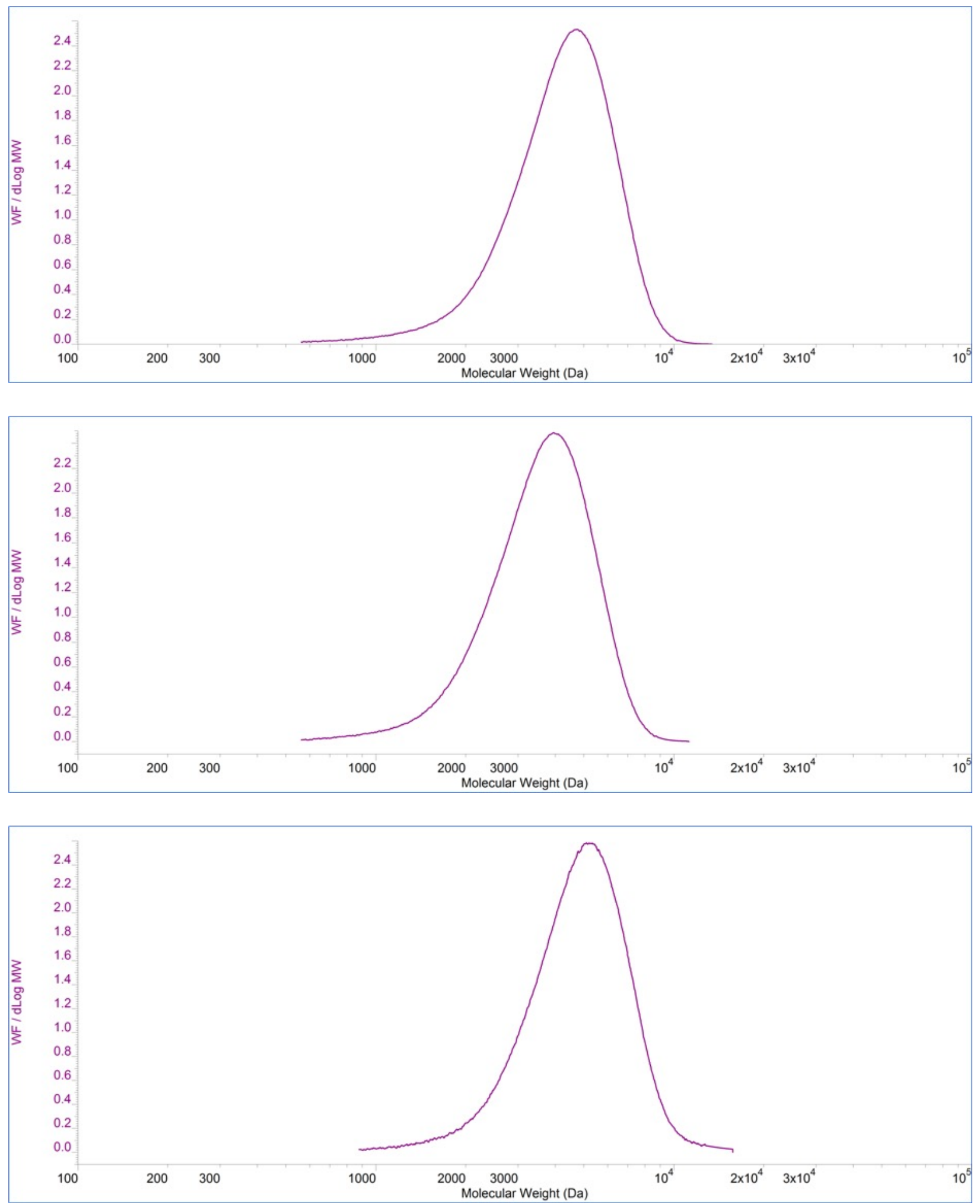

Figure S14. GPC trace of aPH from run 4 (top). GPC trace of aPH from run 5 (middle). GPC trace of aPH from run 6 (bottom). 

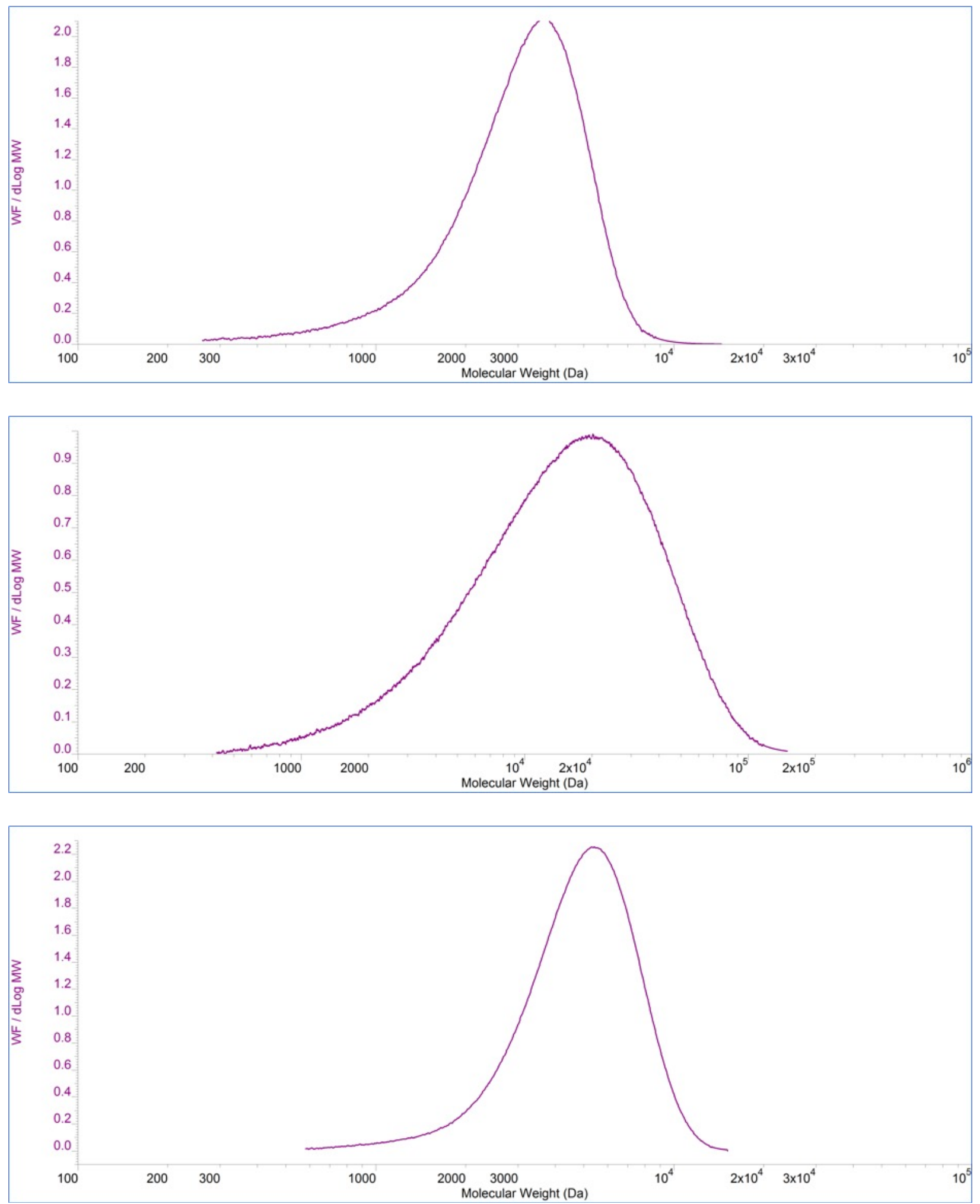

Figure S15. GPC trace of aPH from run 7 (top). GPC trace of aPH from run 8 (middle). GPC trace of aPH from run 9 (bottom). 

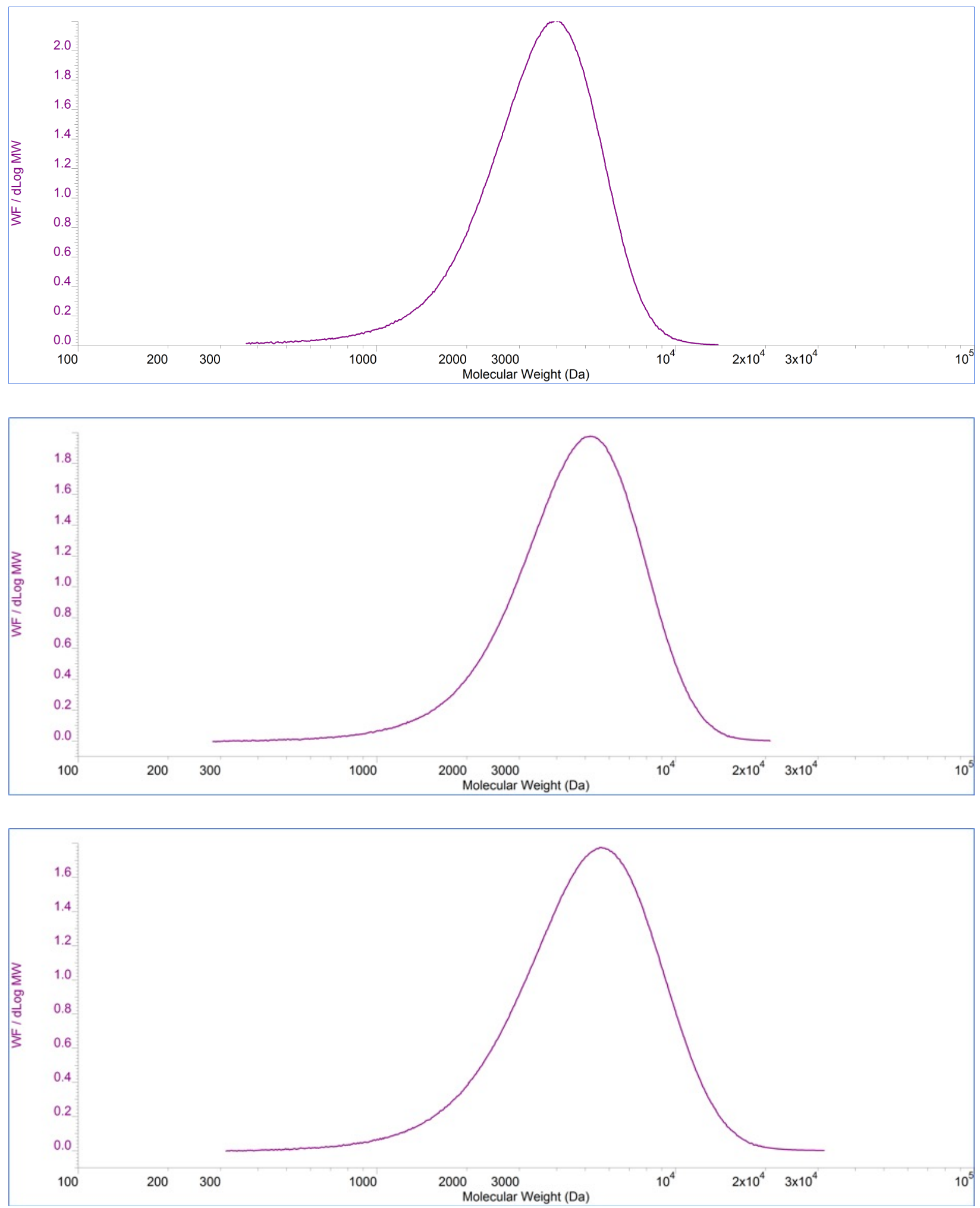

Figure S16. GPC trace of aPH from run 10 (top). GPC trace of aPH from run 11 (middle). GPC trace of aPH from run 12 (bottom). 


\section{$\underline{\text { References }}$}

1. Zhang, W.; Sita, L. R. Highly Efficient, Living Coordinative Chain-Transfer Polymerization of Propene with ZnEt2: Practical Production of Ultrahigh to Very Low Molecular Weight Amorphous Atactic Polypropenes of Extremely Narrow Polydispersity. J. Am. Chem. Soc. 2008, 130, 442-443.

2. Asakura, T.; Demura, M.; Nishiyama, Y. Carbon-13 NMR spectral assignment of five polyolefins determined from the chemical shift calculation and the polymerization mechanism. Macromolecules 1991, 24, 2334-2340.. 